\title{
Seismic Analysis of Earth Slope Using a Novel Sequential Hybrid Optimization Algorithm
}

\author{
Mohammad Khajehzadeh ${ }^{1 *}$, Suraparb Keawsawasvong ${ }^{2}$, Payam Sarir ${ }^{3}$, \\ Dlshad Khurshid Khailany ${ }^{4}$ \\ ${ }^{1}$ Department of Civil Engineering, Anar Branch, Islamic Azad University, 7741943615, Anar, Iran \\ ${ }^{2}$ Department of Civil Engineering, Thammasat School of Engineering, Thammasat University, Pathumthani, Thailand \\ ${ }^{3}$ College of Civil Engineering, Tongji University, Shanghai 200092, China \\ ${ }^{4}$ Department of Civil Engineering, Cihan University- Erbil, Kurdistan Region, Iraq \\ * Corresponding author, e-mail: mohammad.khajehzadeh@anariau.ac.ir
}

Received: 05 October 2021, Accepted: 22 December 2021, Published online: 05 January 2022

\begin{abstract}
One of the most important topics in geotechnical engineering is seismic analysis of the earth slope. In this study, a pseudo-static limit equilibrium approach is applied for the slope stability evaluation under earthquake loading based on the Morgenstern-Price method for the general shape of the slip surface. In this approach, the minimum factor of safety corresponding to the critical failure surface should be investigated and it is a complex optimization problem. This paper proposed an effective sequential hybrid optimization algorithm based on the tunicate swarm algorithm (TSA) and pattern search (PS) for seismic slope stability analysis. The proposed method employs the global search ability of TSA and the local search ability of PS. The performance of the new CTSA-PS algorithm is investigated using a set of benchmark test functions and the results are compared with the standard TSA and some other methods from the literature. In addition, two case studies from the literature are considered to evaluate the efficiency of the proposed CTSA-PS for seismic slope stability analysis. The numerical investigations show that the new approach may provide better optimal solutions and outperform previous methods.
\end{abstract}

\section{Keywords}

soil slope, seismic load, hybrid algorithm, tunicate swarm, pattern search

\section{Introduction}

The assessment of soil slope stability is a well-known problem in civil and geotechnical engineering. In most cases, slope stability analysis is conducted under static loading. However, in the earthquake-prone area the stability assessment of earth slope under seismic loading should be strongly considered. For many years, the limit equilibrium analysis has been used to assess the stability of earthen slopes. In this approach, the factor of safety (FOS) corresponding to the failure surface representing the stability condition of the slope. The Pseudo-Static approach is the commonly used method for seismic slope stability analysis. This approach has been implemented in limit equilibrium methods in which the consequence of earthquake can be determined by an equivalent static force $\left(F_{h}\right)$. The magnitude of this force is a product of a horizontal acceleration coefficient $\left(K_{h}\right)$ and the weight of the potential sliding mass [1].
In a complete slope analysis, the critical failure surface related to the minimum factor of safety should be found amongst all possible trial failure surfaces. This is a complex optimization problem because of the discontinuity of the objective function (i.e., safety factor) and several local minima points available in the search space. Classic deterministic or recent metaheuristic algorithms can be used to solve this problem. Traditional optimization algorithms based on mathematical concepts took a long time or may not obtained the optimum solution at all. To overcome the mentioned problem, during the last few decades, several efficient metaheuristic optimization algorithms have been developed and applied for slope stability evaluation. Some of these research includes: application of genetic algorithm [2], particle swarm optimization [3], simulated annealing and harmony search [4], gravitational search algorithm [5], biogeography-based optimization [6], imperialistic competitive 
algorithm [7] and firefly algorithm [8]. Although metaheuristics methods can produce acceptable results, there is no algorithm that can outperform others in solving all optimization problems. As a result, a number of research have been conducted in order to improve the performance and efficiency of the original algorithms in some aspects and to apply them to a specific application. [9-14]

Tunicate Swarm Algorithm (TSA) is a recently developed bioinspired meta-heuristic optimization technique that is firstly proposed by Kaur et al. [15] in 2020. Tunicates employ swarm intelligence and jet propulsion at sea to find the best state in their environment for finding food. TSA is better than other competitive methods at finding optimal solutions and is suitable for tackling real-world optimization problems. However, it suffers from getting trap in local optima and couldn't converge to a best solution for some complex cases. In order to prevail this drawback, in the current research a sequential hybrid algorithm is developed based on combination of TSA and pattern search method. The proposed hybrid algorithm utilizes the exploration ability of TSA and exploitation ability of PS which can significantly improves the finding results. TSA and pattern search have complementary advantages, and a hybrid of these two algorithms can result in a faster and more robust technique.

\section{Slope Stability Analysis}

One of the important problems of civil engineering is seismic analysis of earth slopes, especially in seismic zones. The stability analysis of earth slope is done through many conventional methods such as limit analysis method, strength reduction method, finite element method and limit equilibrium method. The most commonly applied analytical technique for geotechnical problems is limit equilibrium [16], which evaluates the factor of safety (FOS) based on the Mohr's coulomb criteria. The stability of slope can be determined as the ratio of the available shear strength of the soil to the minimum shear strength required to maintain stability. Several methods of analysis are available based on the limit equilibrium method of slices which are well reviewed and summarized by Duncan [17] and Fredlund and Krahn [18]. The simplified methods are applicable to a specific shape of slip surface such as ordinary method of slices [19] and Bishop method [20], while the rigorous methods are applicable to general shape of failure surface like Spencer [21] and Morgenstern and Price method [22]. Evaluation of accurate behavior of earth slope will be more complicated while seismic loads are applied. Therefore, an effective pseudo-static approach can be utilized to determine the stability of the earth slope under earthquake loads. In this research the effective Morgenstern and Price [22] method of slices along with pseudo-static approach have been adjusted for seismic slope stability analysis.

\subsection{Modeling the factor of safety}

Morgenstern and Price (M-P) developed a comprehensive and rigorous method that satisfies both the force and the moment equilibrium for general form of failure surfaces [22].

In order to consider the seismic load in the pseudo-static analysis, an inertial force $\left(F_{h}\right)$, which is proportional to the weight of slope $(W)$ by factor $K_{h}$, is applied at the center of each slice in horizontal direction and can be defined as:

$F_{h}=\left(W \times a_{h} / g\right)=W \times K_{h}$,

where, $a_{h}$ is the horizontal ground acceleration, $g$ is the acceleration of Earth's gravity and $K_{h}$ is the horizontal acceleration coefficient. In this study, the factor of safety under seismic load is evaluated using the M-P method. Same as other limit equilibrium techniques, in the M-P method, slippery mass will be divided into the number of vertical segments. Let's consider a cross section of a slope with general shape of slip surface and the forces acting on a typical slice as shown in Fig. 1. For the $i$ th slice in this figure, $T_{i}$ is the shear inter-slice force; $E_{i}$ is the normal inter-slice force; $W_{i}$ is the weight of the slice; $N_{i}{ }^{\prime}$ is the effective normal force; $S_{i}$ is the mobilized shear strength; $U_{i}$ is the pore water pressure; $\alpha_{i}$ is the inclination of the slice base, $Q_{i}$ is the external surcharge load, $\delta_{i}$ denotes the inclination of the surcharge load, $h_{i}$ is the average height of slice $i, h_{a}$ is the height of the center of the slice and $F_{h}$ is the horizontal seismic force.

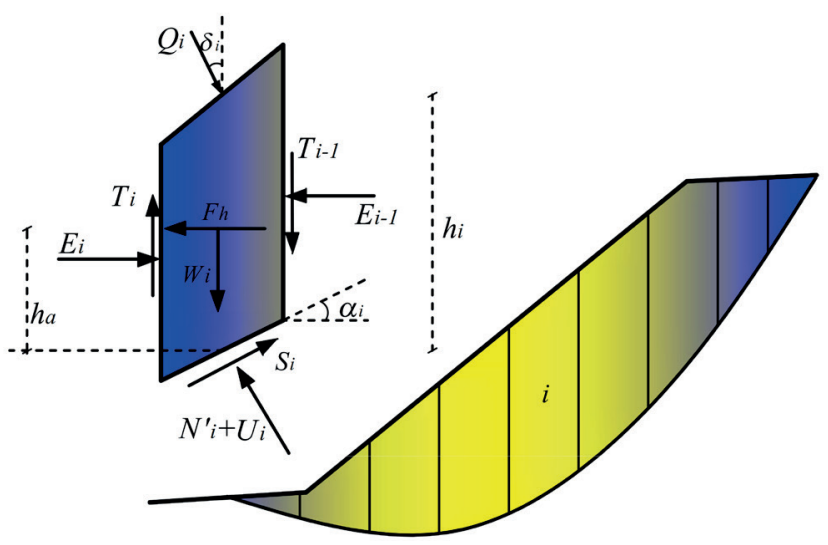

Fig. 1 Forces acting on a typical slice 
In this method, two equations are derived from vertical force and moment equilibrium that include two unknowns: the safety factor and the scaling factor $\lambda$ [22]. Unfortunately, solving for FOS and $\lambda$ is often complex, since the equilibrium equations are highly nonlinear and in complicated form. In order to overcome to the mentioned difficulties, a concise algorithm of $\mathrm{M}-\mathrm{P}$ method developed by Zhu et al. [16] is utilized. In this method, the inclination of the resultant inter-slice force varies symmetrically across the slide mass and the relationship between the normal and shear inter-slice force is expressed as:

$T=f(x) \cdot \lambda \cdot E$,

where, $\lambda$ is a scaling factor and $f(x)$ is the assumed interslice force function. $T$ and $E$ are normal and shear interslice force, respectively as shown in Fig. 1.

The step by step procedure of FOS evaluation is presented in the following:

step 1. Generate a trial slip surface according to the presented procedure in Section 2.2 and divide it to $n$ vertical segments.

step 2. Evaluate $R_{i}$ and $T_{i}$ using the following equations:

$$
\begin{aligned}
R_{i}= & {\left[W_{i} \cos \alpha_{i}-F_{h} \sin \alpha_{i}+Q_{i} \cos \left(\delta_{i}-\alpha_{i}\right)-U_{i}\right] } \\
& \times \tan \varnothing_{i}^{\prime}+c_{i}^{\prime} b_{i} \sec \alpha_{i}, \\
T_{i}= & W_{i} \sin \alpha_{i}+F_{h} \cos \alpha_{i}-Q_{i} \sin \left(\delta_{i}-\alpha_{i}\right),
\end{aligned}
$$

where, $\phi_{i}{ }^{\prime}$ is the effective angle of internal friction at the base of slice $i, c_{i}{ }^{\prime}$ is the effective cohesion at the base of slice $i$, and $b_{i}$ is the width of slice $i$ and. The other parameters are defined in Fig. 1.

step 3. Select inter slices forces function. In this study a constant inter-slice force function $(f(x)=1)$ is considered. step 4. Choose initial values of FOS and $\lambda$ (scaling factor) based on the following criteria:

$$
\text { FOS }>-\frac{\sin \alpha_{i}-\lambda f_{i} \cos \alpha_{i}}{\cos \alpha_{i}+\lambda f_{i} \sin \alpha_{i}} \tan \varnothing^{\prime} .
$$

The appropriate initial values of the FOS and $\lambda$ are 1 and 0 , respectively [16].

step 5. Calculate $\Phi_{i}$ and $\psi_{i-1}$ using Eqs. (6) and (7).

$$
\begin{aligned}
\Phi_{i} & =\left(\sin \alpha_{i}-\lambda f_{i} \cos \alpha_{i}\right) \tan \varnothing_{i}^{\prime} \\
& +\left(\cos \alpha_{i}+\lambda f_{i} \sin \alpha_{i}\right) \times \mathrm{FOS} \\
\Psi_{i-1} & =\left[\begin{array}{c}
\left(\sin \alpha_{i}-\lambda f_{i-1} \cos \alpha_{i}\right) \tan \varnothing_{i}^{\prime}+ \\
\left(\cos \alpha_{i}+\lambda f_{i-1} \sin \alpha_{i}\right) \times F O S
\end{array}\right] / \Phi_{i-1}
\end{aligned}
$$

step 6. Compute FOS using Eq. (8).
$\mathrm{FOS}=\frac{\sum_{i=1}^{n-1}\left(R_{i} \prod_{j=i}^{n-1} \psi_{j}\right)+R_{n}}{\sum_{i=1}^{n-1}\left(T_{i} \prod_{j=i}^{n-1} \psi_{j}\right)+T_{n}}$

step 7. Evaluate $\Phi_{i}$ and $\psi_{i-1}$ and compute FOS again by repeating steps 5 and 6 .

step 8. Determine $E_{i}$ and $\lambda$ according to the following equations.

$$
\begin{aligned}
& E_{i} \Phi_{i}=\Psi_{i-1} E_{i-1} \Phi_{i-1}+\mathrm{FOS} \times T_{i}-R_{i} \\
& \lambda=\frac{\sum\left[b_{i}\left(E_{i}+E_{i-1}\right) \tan \alpha_{i}+F_{h} h_{i}+2 Q \sin \delta_{i} h_{i}\right]}{\sum\left[b_{i}\left(f_{i} E_{i}+f_{i-1} E_{i-1}\right)\right]}
\end{aligned}
$$

step 9. Recalculate FOS with the computed $\lambda$ and the iterative procedure is completed when the difference between the computed FOS and $\lambda$ are less than 0.005 and 0.01 , respectively.

\subsection{Formulation of slip surface}

Generally, in the method of slices, the potential predefined sliding mass is subdivided into a number of vertical segments. Then a critical slip surface associated with the minimum FOS will be searched. To find the most critical slip surface, it is required to produce a proper slip surface and accordingly a trial failure surface generation algorithm is required. In the current research, the slip surface generation algorithm proposed by Cheng [23] is adopted for the analysis. For a slope in Cartesian system, it is necessary to determine the constitutive points of slip surface. The slip surface generating procedure of an arbitrary shape is shown in a Cartesian $\mathrm{X}-\mathrm{Y}$ plane in Fig. 2. In this figure $y=T(\boldsymbol{x})$ describes the geometry of the slope, $y=S(\boldsymbol{x})$ represents the slip surface and $y=R(\boldsymbol{x})$ presents the bedrock line. $x_{i}$ and $y_{i}$ denote the coordinates of the slip surface' segments.

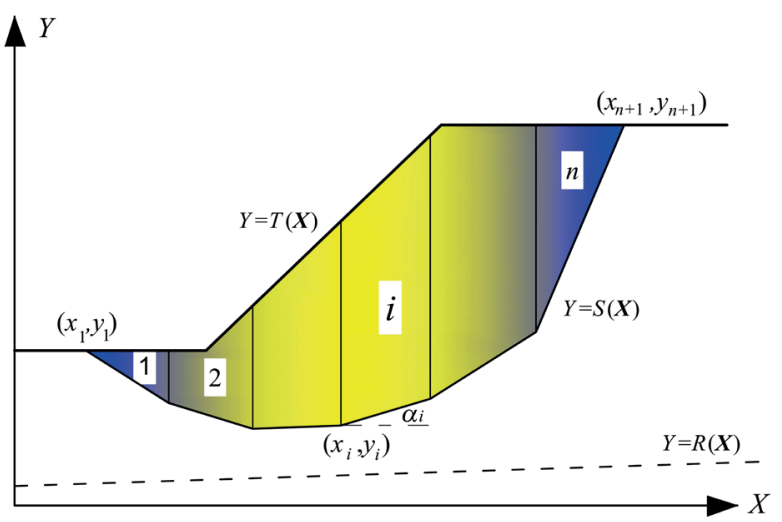

Fig. 2 Procedure for generating acceptable failure surface 
The first step to generate failure surface is dividing the failure soil mass into $n$-vertical segments. The slip surface can be represented by vertices as follows:

$\boldsymbol{V}=\left[x_{1}, y_{1}, x_{2}, y_{2}, \ldots, x_{n}, y_{n}, x_{n+1}, y_{n+1}\right]$.

In order to reduce the number of variables, the width of all the slices is considered to be equal that is obtained using the following equation:

$x_{i+1}=x_{i}+\frac{x_{n+1}-x_{1}}{n} \times(i-1)$.

The upper and lower bounds to the $y$-coordinates $\left(y_{i, \max }\right.$ and $\left.y_{i, \text { min }}\right)$ can be obtained by utilizing the geometry of the slope $(T)$ and the bedrock line $(R)$.

In addition, the trial slip surfaces have to be concave upward. This requirement can be formulated as follows:

$\alpha_{1} \leq \alpha_{2} \leq \ldots \leq \alpha_{i} \leq \ldots \leq \alpha_{n}$,

where $\alpha_{i}$ is the inclination of the base of the slice $i$.

\section{Proposed Hybrid Algorithm}

\subsection{Chaotic tunicate swarm algorithm (CTSA)}

Tunicate swarm algorithm (TSA) is a relatively simple bioinspired meta-heuristic optimization technique inspiration by the swarming behaviors of the marine tunicates and their jet propulsions during its navigation and foraging procedure [15]. This animal has a millimeter-scale form. Tunicate has an ability to find the location of food source in sea. However, there is no idea about the food source in the given search space. When traveling with a jet propulsion behavior, a tunicate must meet three fundamental conditions: (i) it must avoid confrontation with other tunicates in the search space, (ii) it must take the right path to the best search location, and (iii) it must get as close as possible to the best search agent. In TSA, a population of tunicates is swarming in order to search for the best source of food, which represents the fitness function. In this swarming, the tunicates updating their positions related to the first best tunicates that are stored and upgraded in each iteration. The TSA begins with the population of randomly generated tunicates considering the permissible bounds of the design variables according to the following equation:

$\boldsymbol{T}_{p}=\boldsymbol{T}_{p}^{\min }+\operatorname{rand} \times\left(\boldsymbol{T}_{p}^{\max }-\boldsymbol{T}_{p}^{\min }\right)$,

where, $\boldsymbol{T}_{p}$ is the position of each tunicate and rand is a random number within range $[0,1] . \boldsymbol{T}_{p}^{\min }$ and $\boldsymbol{T}_{p}^{\max }$ are minimum and maximum values of design variables, respectively. During the iterations, the tunicates update their position through the following formula [15]:
$\boldsymbol{T}_{p}(\boldsymbol{x}+1)=\frac{\boldsymbol{T}_{p}(x)+\boldsymbol{T}_{p}(\boldsymbol{x}+1)}{2+c_{1}}$

where, $c_{1}$ is a random number within range $[0,1]$ and $\boldsymbol{T}_{p}$ refers to the updated position of the tunicate with respect to the position of the food source based on Eq. (16).

$$
\boldsymbol{T}_{p}(x)=\left\{\begin{array}{l}
S F+A \times \mid S F-\text { rand } \times \boldsymbol{T}_{p} \mid, \text { if rand } \geq 0.5 \\
S F-A \times \mid S F-\text { rand } \times \boldsymbol{T}_{p} \mid, \text { if rand }<0.5
\end{array},\right.
$$

where, $S F$ is the source of food which is represented by the best tunicate position in the whole population; $\boldsymbol{A}$ is a randomized vector to prevent confrontation of tunicates with each other which is modelled as:

$$
\boldsymbol{A}=\frac{c_{2}+c_{3}-2 c_{1}}{V T_{\min }+c_{1}\left(V T_{\max }-V T_{\min }\right)},
$$

where, $c_{1}, c_{2}$ and $c_{3}$ are random numbers within range $[0,1]$; $V T_{\min }$ and $V T_{\max }$ represent the premier and subordinate speeds to produce social interaction which considered as 1 and 4, respectively [15].

The aim of the current research, is implementation of the global search ability of the TSA. To this aim and to increase the exploration ability of the algorithm, the chaotic sequence is applied in the tunicate updating position equation (Eq. (16)). Chaotic systems are deterministic systems that presents randomness, irregularity and the stochastic property depend on the initial conditions. Chaotic variables can oscillate through a certain ranges based on their own irregularity without repetition. A chaotic map is a map that presents some kind of chaotic behavior with capability of generating chaotic motion. In the current study, the well-known logistic map is applied based on the following equation:

$\lambda(x+1)=a \times \lambda(x) \times(1-\lambda(x))$.

In this equation, $\lambda(x)$ is the chaotic map and $\mathrm{x}$ denoting the iteration number. $\lambda(0)$ is in the range of $(0,1)$ and should not be equal to $0,0.25,0.5,0.75$ and $1 . a$ is a constant equal to 4 . In the chaotic TSA (CTSA), the randomized parameter $(A)$ in Eq. (16) multiplied by $\lambda$ to increase the stochastic behavior of the algorithm and avoiding premature convergence. Therefore, the updated position of the tunicate with respect to the position of the food source is evaluated using the following equation:

$\boldsymbol{T}_{p}(x)=\left\{\begin{array}{l}S F+\lambda \times A \times \mid S F-\text { rand } \times \boldsymbol{T}_{p} \mid, \text { if rand } \geq 0.5 \\ S F-\lambda \times A \times \mid S F-\text { rand } \times \boldsymbol{T}_{p} \mid, \text { if rand }<0.5\end{array}\right.$ 
The steps of the proposed CTSA are given below.

Step 1: Initialize the tunicate population $\boldsymbol{T}_{p}$ based on Eq. (14). Step 2: Choose the initial parameters and maximum number of iterations.

Step 3: Calculate the fitness value of each search agent.

Step 4: The best tunicate is explored in the given search space.

Step 5: Update the position of each tunicate using Eq. (15). Step 6: Adjust the updated tunicate which goes beyond the boundary in a given search space.

Step 7: Compute the updated tunicate fitness value. If there is a better solution than the previous optimal solution, then update the best.

Step 8: If the stopping criterion is satisfied, then the algorithm stops. Otherwise, repeat the Steps 5-8.

Step 9: Return the best optimal solution which is obtained so far.

\subsection{Pattern search (PS)}

PS is a derivative-free algorithm that can be simply implemented to fine-tune local search. The PS algorithm generates a set of points that may or may not be close to the optimum [24]. In the first iteration, a mesh (a collection of points) is created around an existing point. If a new point in the mesh has a lower value of objective function, it becomes the current point in the following iteration.

The PS starts the search with an initial point $X_{0}$ defined by the user. At the first iteration, the mesh size is considered equal to 1 and the pattern vectors (or direction vectors) are constructed as $\left[\begin{array}{ll}0 & 1\end{array}\right]+X_{0},\left[\begin{array}{ll}1 & 0\end{array}\right]+X_{0},\left[\begin{array}{ll}-1 & 0\end{array}\right]+X_{0}$ and $[0-1]+X_{0}$, and new mesh points are added as presented in Fig. 3. Then, the objective function is calculated for produced trial points until a value smaller than $\mathrm{X} 0$ is found. If there is such a point $\left(f\left(X_{1}\right)<f\left(X_{0}\right)\right)$, the poll is successful and the algorithm sets this point as source point. After a successful poll, the algorithm multiplies the current

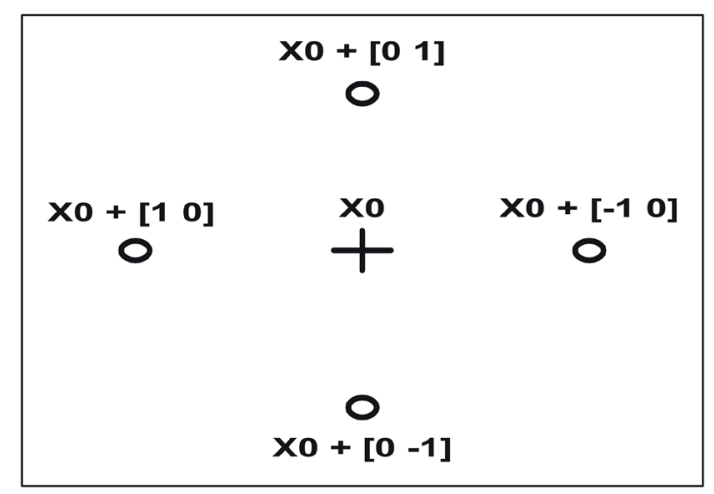

Fig. 3 Pattern Search mesh points with pattern mesh size by 2 , (called the expansion factor) and proceeds to iteration 2 with the following new points: $2 \times\left[\begin{array}{ll}0 & 1\end{array}\right]+X_{1}$, $2 \times\left[\begin{array}{ll}1 & 0\end{array}\right]+X_{1}, 2 \times[-10]+X_{1}$ and $2 \times[0-1]+X_{1}$, if a value smaller than for $X_{1}$ is found, $X_{2}$ is defined, the mesh size is increased by two and iterations continue. If at any stage the poll is unsuccessful (i.e., no point has an objective function smaller than the most recent value) the current point is not changed and the mesh size is reduced by multiplying by a contraction factor. These processes repeated until the minimum is found or a terminating condition is met.

\subsection{Hybrid CTSA-PS}

A hybrid algorithm is an algorithm that combines two or more algorithms for solving a same problem. Hybridization aims to combine the advantages of each algorithm to increase the accuracy of the result. There exist several types of hybridization such as sequential, parallel, and integrative ones. The sequential hybridization is the most widely used method. It consists of applying several methods in such a manner that the results of a given method are taken as initial solutions to the next method [25].

In this article, sequential hybridization, which consists of the combination of both the CTSA and PS algorithms, referred to as CTSA-PS is proposed for seismic slope stability analysis. The hybrid algorithm can use of not only the strong global searching ability of the CTSA, but also the strong local searching ability of the PS algorithm. Chaotic tunicate swarm algorithm has good global optimal performance and is easy to jump out of local minima. In theory, increasing the iteration numbers of CTSA can improve the search accuracy. However, when the iteration numbers are enough large, CTSA cannot improve the precision. So the local search ability of CTSA is still insufficient. Pattern search is a local optimization method and the initial point has great influence on the algorithm's results and different initial points will cause a large difference in the results. But pattern search will be a simple and effective method if a good initial point is selected. In this paper, we combine the advantages of the CTSA as global optimization and pattern search as local optimization to find the optimal solution, effectively. The proposed hybrid algorithm begins with the CTSA since the PS is sensitive to the initial solution. The searching process continues with the CTSA for a specific number of iterations. Then, the PS is activated to perform a local search using the current best solution obtained by CTSA as its starting point. The flowchart of the proposed hybrid algorithm is depicted in Fig. 4. 


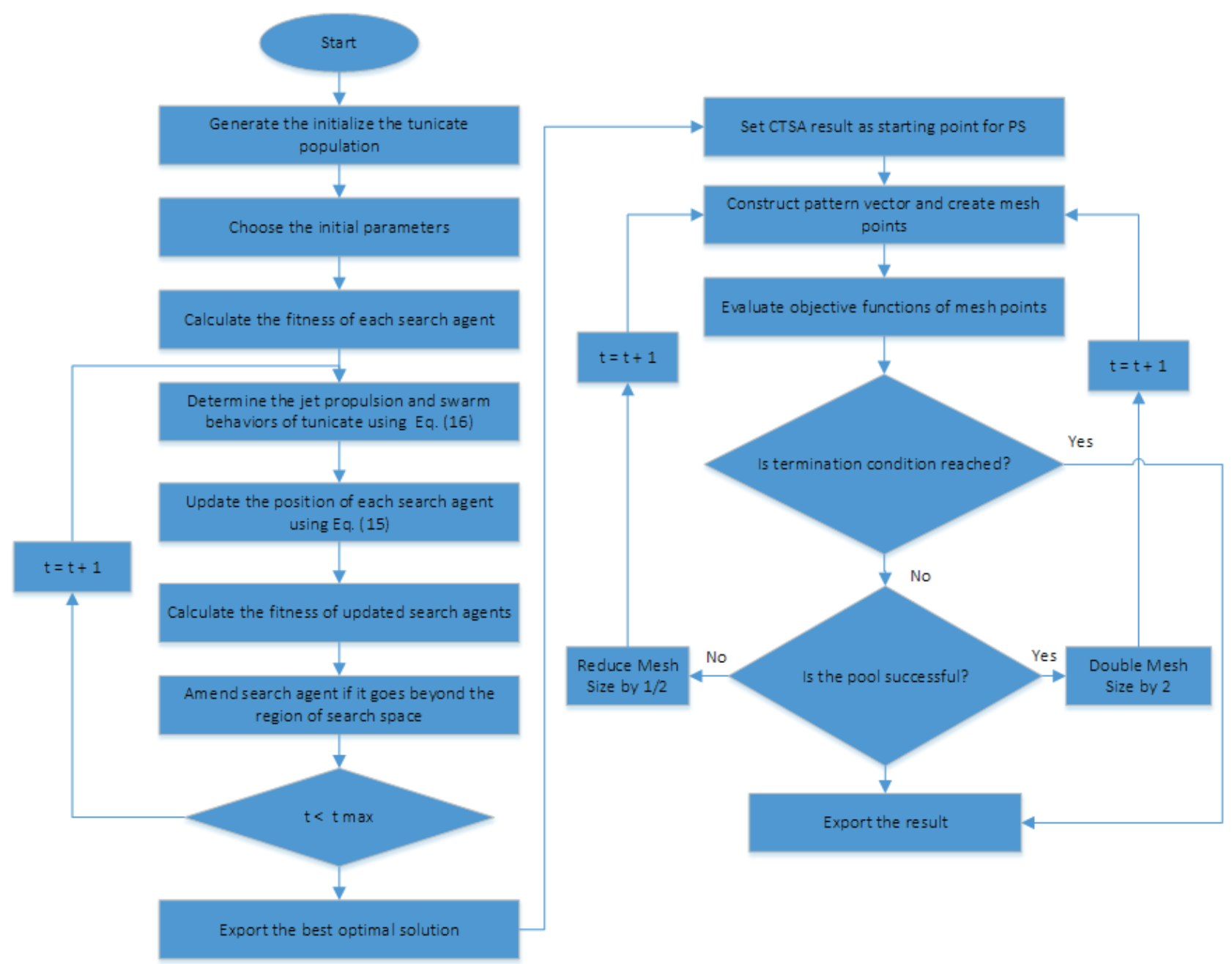

Fig. 4 Flowchart of the CTSA-PS

\section{Comparative Analysis of the CTSA-PS}

In this section the effectiveness verification of the proposed method will be investigated. To this aim, the performance of CTSA-PS is compared with the standard version of the algorithm as well as some well-known metaheuristic algorithms on a collection of benchmark functions from the literature. These are all minimization problems that can be used to assess the robustness and search efficiency of new optimization algorithms. Table 1 shows the mathematical formulation and features of these test functions. The results and performance of the proposed CTSA-PS is compared with TSA and other well-established optimization algorithms include Sine-Cosine Algorithm (SCA) [26], and Grey Wolf Optimizer (GWO) [27]. These algorithms have been proved their effectiveness and robustness in compared with other well-established methods like Particle Swarm Optimization, Genetic Algorithm, Firefly Algorithm and so on $[26,27]$. For both CTSA-PS and TSA the number of tunicate $(N)$ is considered as 80 and the maximum number of iteration is equal to 1000 . Because metaheuristics approaches are stochastic, the findings of a single run may be erroneous, and the algorithms may find better or worse solutions than those previously found. As a result, statistical analysis should be used to make a fair comparison and evaluate the algorithms' effectiveness. In order to address this issue, 30 separate runs are done for the specified algorithms, and statistical results are collected and reported in Table 2.

The results of Table 2 show that, for all functions except $F_{6}$, CTSA-PS could provide better results, which means that the new algorithm has a large potential search space compared with the standard TSA and also other optimization algorithms. From the standard deviation point of view, which indicates the stability of the algorithm, the results show that CTSA-PS is a more stable method when compared with the other techniques. From the obtained results, it can be concluded that CTSA-PS outperforms the standard algorithm and other methods. 
Table 1 Description of benchmark functions

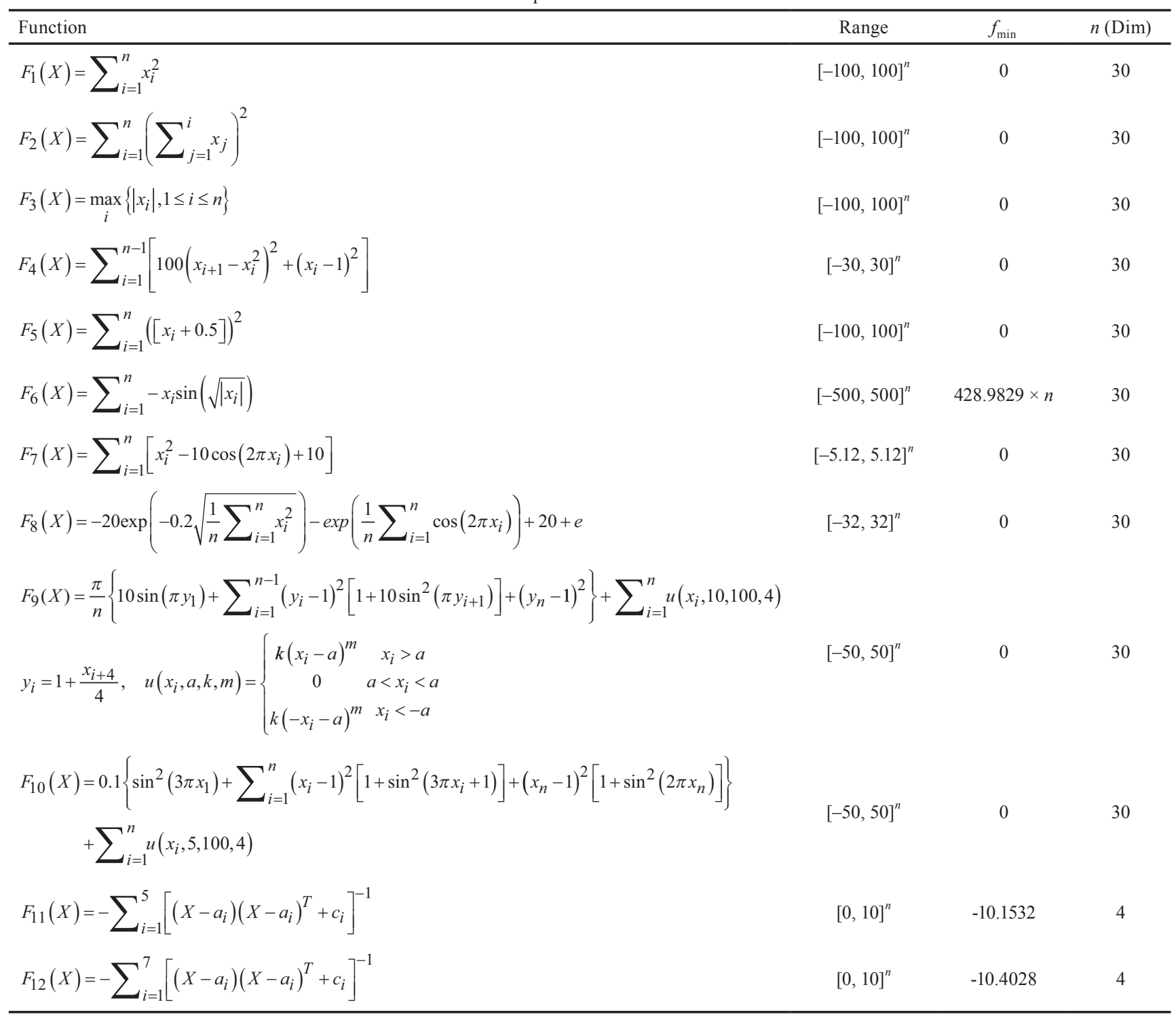

\section{Model application}

Based on the main objective of the current study, in this section the proposed CTSA-PS algorithm is applied for seismic slope stability evaluation problems. The factor of safety under static and seismic loads has been calculated using M-P method for general shape of slip surface. The critical slip surface associated with the minimum factor of safety is evaluated using the proposed CTSA-PS algorithm. The applicability and effectiveness of the proposed methodology in searching the optimum value of FOS (i.e., solution of objective function), two numerical experiments are considered from the previous studies. For both problems, number of slices of the predefined failure surface is considered equal to 40 . Both cases are solved by considering three different values of $K_{h}$ which are equal to $0.0,0.1$ and 0.2 . Owing to the stochastic behavior of the CTSA-PS, the algorithm is run 30 times independently for each loading condition and the best results are reported.

\subsection{Test problem 1: slope in a homogeneous soil}

The first problem is taken from the study of Zolfaghari et al. [2] which is a dry slope in a homogeneous soil. The geometric layout for the soil slope is shown in Fig. 5. The total height of the slope is $10.0 \mathrm{~m}$ and the slope angle is $26.56^{\circ}$. The corresponding geotechnical parameters of the slope are: effective friction angle $\left(\phi^{\prime}\right)$ equal to $20^{\circ}$, effective cohesion intercept $\left(c^{\prime}\right)$ equal to $14.71 \mathrm{kPa}$ and unit weight $(\gamma)$ is equal to $18.63 \mathrm{kN} / \mathrm{m}^{3}$. 
Table 2 Comparison of different methods in solving test functions

\begin{tabular}{|c|c|c|c|c|c|}
\hline Function & Statistics & CTSA-PS & TSA & SCA & GWO \\
\hline$F_{1}$ & $\begin{array}{l}\text { Best } \\
\text { Mean } \\
\text { Std. }\end{array}$ & $\begin{array}{l}0.00 \\
0.00 \\
0.00\end{array}$ & $\begin{array}{l}5.1458 \mathrm{e}-61 \\
8.3155 \mathrm{e}-56 \\
2.4905 \mathrm{e}-55\end{array}$ & $\begin{array}{l}1.5523 \mathrm{e}-07 \\
2.3458 \mathrm{e}-04 \\
7.9295 \mathrm{e}-04\end{array}$ & $\begin{array}{l}2.4915 \mathrm{e}-61 \\
4.9162 \mathrm{e}-59 \\
1.0230 \mathrm{e}-58\end{array}$ \\
\hline$F_{2}$ & $\begin{array}{l}\text { Best } \\
\text { Mean } \\
\text { Std. }\end{array}$ & $\begin{array}{l}3.5412 \mathrm{e}-52 \\
1.0214 \mathrm{e}-42 \\
2.8261 \mathrm{e}-42\end{array}$ & $\begin{array}{c}2.5684 \mathrm{e}-32 \\
8.1741 \mathrm{e}-19 \\
4.4714 \mathrm{e}-18\end{array}$ & $\begin{array}{c}70.8285 \\
789.1620 \\
746.2287\end{array}$ & $\begin{array}{l}1.2533 \mathrm{e}-19 \\
1.5096 \mathrm{e}-14 \\
6.5547 \mathrm{e}-14\end{array}$ \\
\hline$F_{3}$ & $\begin{array}{c}\text { Best } \\
\text { Mean } \\
\text { Std. }\end{array}$ & $\begin{array}{l}3.7058 \mathrm{e}-21 \\
1.4598 \mathrm{e}-19 \\
2.0348 \mathrm{e}-19\end{array}$ & $\begin{array}{l}3.2458 \mathrm{e}-08 \\
1.0102 \mathrm{e}-05 \\
1.6927 \mathrm{e}-05\end{array}$ & $\begin{array}{l}1.2610 \\
9.3080 \\
8.0720\end{array}$ & $\begin{array}{l}9.8174 \mathrm{e}-16 \\
1.9487 \mathrm{e}-14 \\
4.4955 \mathrm{e}-14\end{array}$ \\
\hline$F_{4}$ & $\begin{array}{l}\text { Best } \\
\text { Mean } \\
\text { Std. }\end{array}$ & $\begin{array}{l}8.8638 \\
9.7268 \\
0.4949\end{array}$ & $\begin{array}{c}25.6273 \\
28.4422 \\
0.7616\end{array}$ & $\begin{array}{c}27.3230 \\
29.9106 \\
4.1508\end{array}$ & $\begin{array}{c}25.2273 \\
26.9256 \\
0.8418\end{array}$ \\
\hline$F_{5}$ & $\begin{array}{l}\text { Best } \\
\text { Mean } \\
\text { Std. }\end{array}$ & $\begin{array}{l}0.00 \\
0.00 \\
0.00\end{array}$ & $\begin{array}{l}2.0585 \\
3.6724 \\
0.6918\end{array}$ & $\begin{array}{l}3.4070 \\
4.0360 \\
0.2954\end{array}$ & $\begin{array}{l}0.2466 \\
0.6376 \\
0.3353\end{array}$ \\
\hline$F_{6}$ & $\begin{array}{l}\text { Best } \\
\text { Mean } \\
\text { Std. }\end{array}$ & $\begin{array}{c}-1.1148 \mathrm{e}+04 \\
-1.0631 \mathrm{e}+04 \\
296.9742\end{array}$ & $\begin{array}{c}-7.8992 \mathrm{e}+03 \\
-6.6126 \mathrm{e}+03 \\
599.2609\end{array}$ & $\begin{array}{c}-5.2993 \mathrm{e}+03 \\
-4.0769 \mathrm{e}+03 \\
336.8249\end{array}$ & $\begin{array}{c}-8.8178 \mathrm{e}+03 \\
-6.2524 \mathrm{e}+03 \\
852.4634\end{array}$ \\
\hline$F_{7}$ & $\begin{array}{l}\text { Best } \\
\text { Mean } \\
\text { Std. }\end{array}$ & $\begin{array}{l}1.1369 \mathrm{e}-13 \\
3.2969 \mathrm{e}-13 \\
1.7116 \mathrm{e}-13\end{array}$ & $\begin{array}{c}77.7761 \\
151.4539 \\
35.8717\end{array}$ & $\begin{array}{c}1.0560 \mathrm{e}-06 \\
5.9694 \\
12.2476\end{array}$ & $\begin{array}{c}0.00 \\
0.8853 \\
2.4438\end{array}$ \\
\hline$F_{8}$ & $\begin{array}{l}\text { Best } \\
\text { Mean } \\
\text { Std. }\end{array}$ & $\begin{array}{l}1.5099 \mathrm{e}-14 \\
5.2758 \mathrm{e}-14 \\
2.1591 \mathrm{e}-14\end{array}$ & $\begin{array}{c}1.5099 \mathrm{e}-14 \\
2.4095 \\
1.3920\end{array}$ & $\begin{array}{c}1.5579 \mathrm{e}-05 \\
14.3622 \\
8.9778\end{array}$ & $\begin{array}{l}1.1546 \mathrm{e}-14 \\
1.5928 \mathrm{e}-14 \\
2.5861 \mathrm{e}-15\end{array}$ \\
\hline$F_{9}$ & $\begin{array}{l}\text { Best } \\
\text { Mean } \\
\text { Std. }\end{array}$ & $\begin{array}{l}1.5705 e-32 \\
1.5705 e-32 \\
2.8850 e-48\end{array}$ & $\begin{array}{l}0.2738 \\
6.3735 \\
3.4586\end{array}$ & $\begin{array}{l}0.2631 \\
0.9568 \\
1.1497\end{array}$ & $\begin{array}{l}0.0121 \\
0.0364 \\
0.0201\end{array}$ \\
\hline$F_{10}$ & $\begin{array}{l}\text { Best } \\
\text { Mean } \\
\text { Std. }\end{array}$ & $\begin{array}{c}1.3498 \mathrm{e}-32 \\
0.0099 \\
0.0035\end{array}$ & $\begin{array}{l}1.7796 \\
2.8976 \\
0.6436\end{array}$ & $\begin{array}{l}1.8452 \\
3.4211 \\
3.9911\end{array}$ & $\begin{array}{l}0.1006 \\
0.5280 \\
0.2359\end{array}$ \\
\hline$F_{11}$ & $\begin{array}{l}\text { Best } \\
\text { Mean } \\
\text { Std. }\end{array}$ & $\begin{array}{c}-10.1532 \\
-9.3918 \\
1.2321\end{array}$ & $\begin{array}{c}-10.1361 \\
-7.2879 \\
2.8594\end{array}$ & $\begin{array}{l}-8.1370 \\
-4.3187 \\
2.0785\end{array}$ & $\begin{array}{c}-10.1531 \\
-9.4790 \\
1.7469\end{array}$ \\
\hline$F_{12}$ & $\begin{array}{l}\text { Best } \\
\text { Mean } \\
\text { Std. }\end{array}$ & $\begin{array}{c}-10.4029 \\
-10.1029 \\
0.893\end{array}$ & $\begin{array}{c}-10.3812 \\
-7.8325 \\
3.1843\end{array}$ & $\begin{array}{c}-9.0513 \\
-5.4154 \\
1.7315\end{array}$ & $\begin{array}{c}-10.4029 \\
-10.2253 \\
0.9703\end{array}$ \\
\hline
\end{tabular}

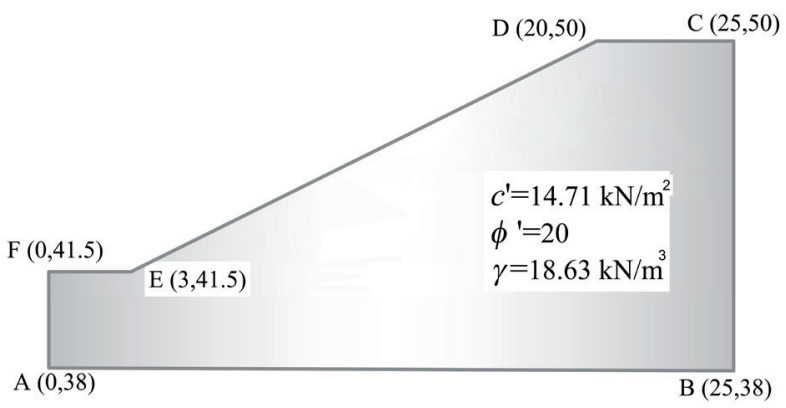

Fig. 5 Homogenous slope of test problem 1

This problem has been solved by Zolfaghari et al [2] using simple genetic algorithm (GA) and Morgenstern and Price method applied to analyses the slope with non-circular failure surface. Cheng et al. [4] have been adopted six heuristic algorithms and the Spencer's method [21] by considering non-circular failure surface for the solution. According to their results, simulated annealing (SA) and harmony search
(HS) algorithm could provide better results compared with other methods [4]. In addition, Himanshu and Burman [28] developed particle swarm optimization along with Bishop's method for the solution. All of these studies solved the problem under static load which is equivalent with $K_{h}=0$ (case 1) in the present study. The problem is solved using the TSA and CTSA-PS algorithms for different values of horizontal acceleration coefficient and the corresponding minimum FOS values are reported in Table 3.

According to the results of Table 3, the minimum FOS evaluated by the proposed CTSA-PS is 1.7155 and it is lower than those obtained by the other methods when $K_{h}$ is equal zero. In addition, the calculated safety factors using the proposed hybrid algorithm is lower than those evaluated by original TSA for all loading cases. The corresponding critical failure surfaces for different value of $K_{h}$ are graphically presented in Fig. 6. 


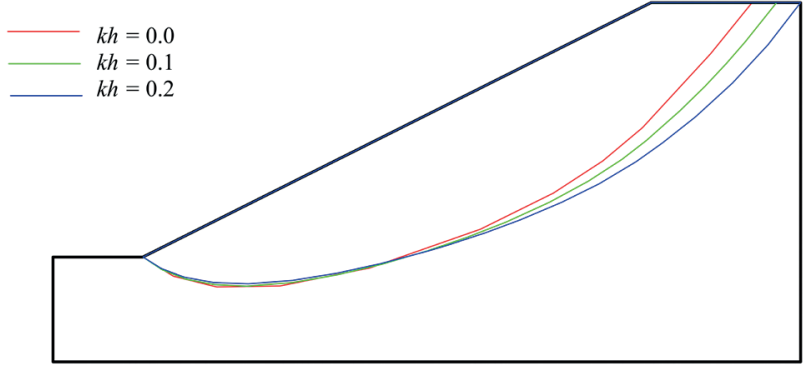

Fig. 6 Critical slip surfaces of test problem 1

Average values of FOS
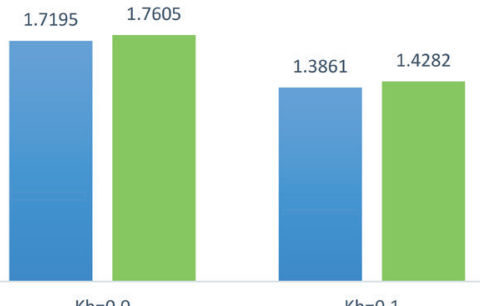

$\mathrm{Kh}=0.1$

$=$ CTSA-PS $=$ TSA

Fig. 7 Average values of FOS for test problem 1

\section{Standard Deviation of FOS}

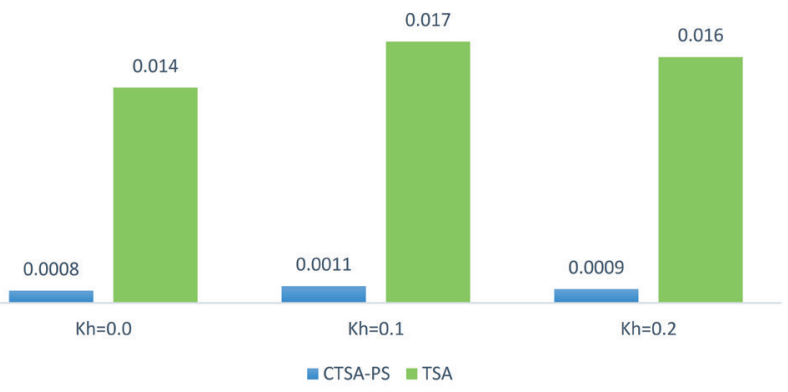

Fig. 8 standard deviation of FOS for test problem 1

The average and standard deviation of the FOS from 30 separate runs are shown in Figs. 7 and 8 respectively. According to these results, the mean values of FOS obtained by CTSA-PS are slightly lower than those of TSA. In addition, the standard deviation of the results of proposed method is much lower than those of standard algorithm, which proves that the CTSA-PS strongly improves the instability of the TSA algorithm. It is found from the above results that CTSA-PS is capable of obtaining a lower value of FOS and the corresponding critical failure surface and this reflects its advantage.

In addition, the obtained results of Table 3 reveals that by increasing horizontal acceleration coefficient to 0.1 and 0.2 , the FOS will be decreased up to $19 \%$ and $32 \%$ respectively. For this problem, a sensitivity analysis has been conducted to investigate the influence of horizontal acceleration coefficient on the minimum FOS.

The horizontal acceleration coefficient is the main factor influencing the safety of a slope under earthquake loading. In this sense, Fig. 9 shows the parabolic curves of minimum factor of safety versus different values of horizontal acceleration coefficient with increments of 0.05 . As shown in this figure, the minimum FOS decrease drastically as the horizontal acceleration coefficient increases. According to the results, the safety factor adjusts to FOS $=4.025 \times K_{h}^{2}-$ $3.655 \times K_{h}+1.709$ with $\mathrm{R}^{2}=0.9997$. Based on this equation, the critical acceleration coefficient with respect to $\mathrm{FOS}=1$, is obtained equal to 0.28 .

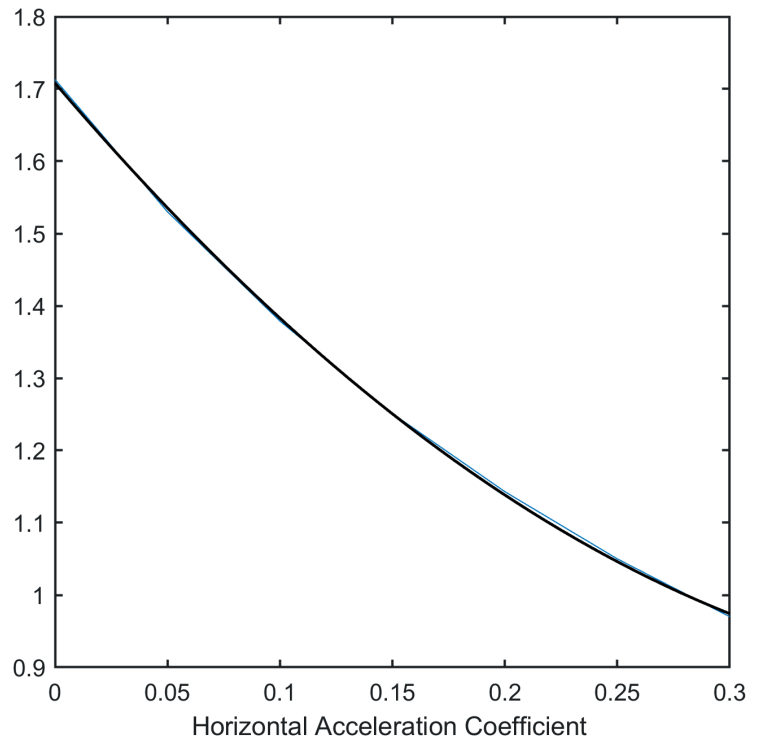

Fig. 9 Effect of $K_{h}$ on FOS

Table 3 Minimum FOS for test problem 1

\begin{tabular}{|c|c|c|c|c|c|}
\hline \multirow{2}{*}{ Optimization method } & \multirow{2}{*}{$\begin{array}{l}\text { Limit equilibrium } \\
\text { method }\end{array}$} & \multirow{2}{*}{ Number of slices } & \multicolumn{3}{|c|}{ Minimum FOS } \\
\hline & & & $K_{h}=0.0$ & $K_{h}=0.1$ & $K_{h}=0.2$ \\
\hline GA [2] & M-P method & - & 1.75 & - & _ \\
\hline $\mathrm{SA}[4]$ & Spencer's method & 40 & 1.7267 & - & - \\
\hline HS [4] & Spencer's method & 40 & 1.7264 & _- & - \\
\hline PSO [28] & Bishop's method & 40 & 1.7195 & - & - \\
\hline TSA (current study) & M-P method & 40 & 1.7275 & 1.4012 & 1.1897 \\
\hline CTSA-PS (current study) & M-P method & 40 & 1.7155 & 1.3821 & 1.1454 \\
\hline
\end{tabular}




\subsection{Test problem 2: slope in a non-homogeneous soil}

The second experiment also taken from the study of Zolfaghri et al [2] which is a natural slope with four different soil layers as shown in Fig. 10. The water surface at elevation of 46.7 is depicted by dashed line.

The geotechnical parameters for this problem are presented in Table 4. Zolfaghari et al. [2] applied genetic algorithm (GA) along with the Morgenstern and Price's method for this problem. Four different loading condition are considered by Zolfaghari et al.[2] include: no water pressure and no earthquake loadings (case 1); water pressure and no earthquake loading (case 2); earthquake loading with $K_{h}=0.1$ and no water pressure (case 3); water pressure and earthquake loading; (case 4). This problem is solve using the proposed CTSA-PS algorithm and in addition to the mentioned loading conditions two more cases are considered. Earthquake loading with $K_{h}=0.2$ and no water pressure (case 5); water pressure and earthquake loading with $K_{h}=0.2$ (case 6).

This problem is also solved by Cheng et al. [4] using six heuristic algorithms and Spencer's method [21] for non-circular failure surface. As reported by Cheng et al. [4], particle swarm optimization (GA) could provide lower value

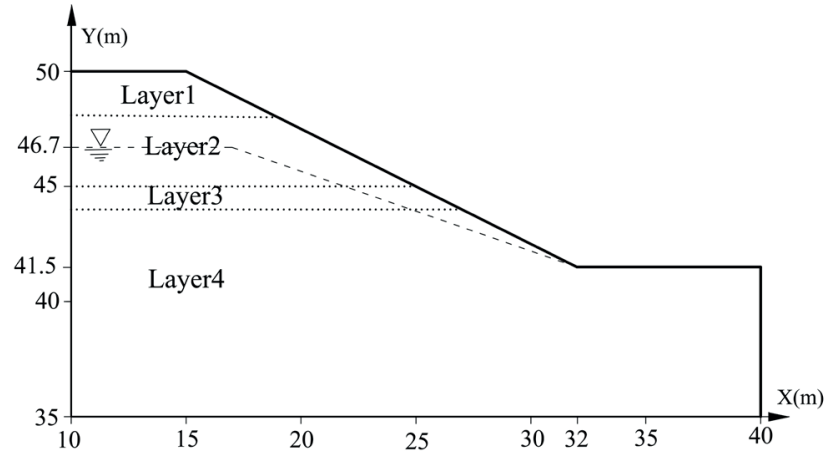

Fig. 10 Non-Homogenous slope of test problem 2

Table 4 Geotechnical parameters for test problem 2

\begin{tabular}{lccc}
\hline Layer & $\gamma\left(\mathrm{kN} / \mathrm{m}^{3}\right)$ & $c^{\prime}(\mathrm{kPa})$ & $\phi^{\prime}(\mathrm{deg})$ \\
\hline 1 & 18.63 & 14.7 & 20 \\
2 & 18.63 & 16.7 & 21 \\
3 & 18.63 & 4.9 & 10 \\
4 & 18.63 & 34.3 & 28 \\
\hline
\end{tabular}

of FOS compared with other techniques. The results of the reported and calculated minimum factor of safety are summarized in Table 5 for six loading conditions.

As per the results of Table 5, the optimum value of the safety factor evaluated using the proposed methodology are much lower than those evaluated by GA and slightly lower than those calculated by PSO for all loading conditions. Figs. 11 and 12 show the average and standard deviation of the FOS from 30 independent runs for different loading condition. From the above results, it may be concluded that the new CTSA-PS algorithm can be applied for seismic slope stability evaluation, effectively. This example also proves that the CTSA-PS algorithm can be applied to slope stability analysis with ground water.

\section{Summery and conclusion}

A hybrid global optimization algorithm called CTSA-PS has been introduced for earth slope stability evaluation under seismic loading. Earthquake is an important force that can cause the failure of slopes in a seismically active

\section{Average values of FOS}

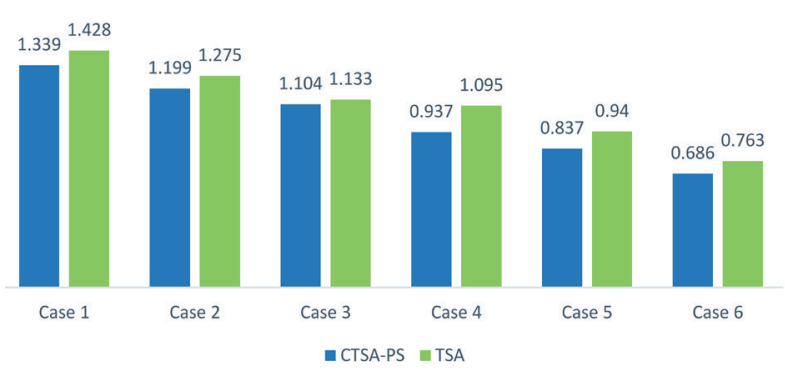

Fig. 11 Average values of FOS for example 2

\section{Standard Deviation of FOS}

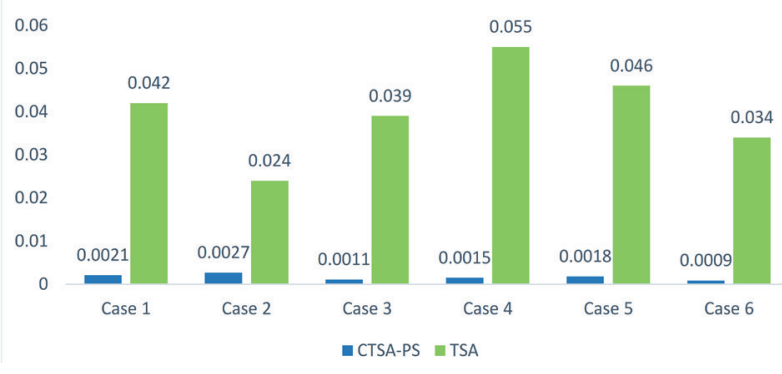

Fig. 12 standard deviation of FOS for example 2

Table 5 Minimum FOS for test problem 2

\begin{tabular}{|c|c|c|c|c|c|c|c|}
\hline Optimization method & Limit equilibrium method & Case 1 & Case2 & Case3 & Case 4 & Case 5 & Case6 \\
\hline GA [2] & M-P method & 1.48 & 1.36 & 1.37 & 0.98 & - & - \\
\hline PSO [4] & Spencer's method & 1.3372 & 1.21 & 1.0474 & 0.9451 & - & - \\
\hline TSA (current study) & M-P method & 1.342 & 1.206 & 1.0546 & 0.985 & 0.849 & 0.69 \\
\hline CTSA-PS (current study) & M-P method & 1.331 & 1.193 & 1.0406 & 0.933 & 0.831 & 0.681 \\
\hline
\end{tabular}


region and to prevent and mitigate the damages, seismic analysis of earth slope is required. The Morgenstern and Price method has been applied to calculate the safety factor for general shape of slip surface and pseudo-static method adopted for the seismic analysis. The proposed CTSA-PS algorithm combines two search techniques: the chaotic TSA as an effective global optimization and pattern search as a robust local search method. The performance of the proposed algorithm is benchmarked using a set of unimodal and multi-modal test functions and the results were compared with TSA and some of the recently

\section{References}

[1] Baker, R., Shukha, R., Operstein, V., Frydman, S. "Stability charts for pseudo-static slope stability analysis", Soil Dynamics and Earthquake Engineering, 26(9), pp. 813-823, 2006. https://doi.org/10.1016/j.soildyn.2006.01.023

[2] Zolfaghari, A. R., Heath, A. C., McCombie, P. F. "Simple genetic algorithm search for critical non-circular failure surface in slope stability analysis", Computers and Geotechnics, 32(3), pp. 139-152, 2005 .

https://doi.org/10.1016/j.compgeo.2005.02.001

[3] Cheng, Y. M., Li, L., Chi, S., Wei, W. B. "Particle swarm optimization algorithm for the location of the critical non-circular failure surface in two-dimensional slope stability analysis", Computers and Geotechnics, 34(2), pp. 92-103, 2007. https://doi.org/10.1016/j.compgeo.2006.10.012

[4] Cheng, Y. M., Li, L., Chi, S. C. "Performance studies on six heuristic global optimization methods in the location of critical slip surface", Computers and Geotechnics, 34(6), pp. 462-484, 2007.

https://doi.org/10.1016/j.compgeo.2007.01.004

[5] Khajehzadeh, M., Taha, M. R., El-Shafie, A., Eslami, M. "Search for critical failure surface in slope stability analysis by gravitational search algorithm", International Journal of Physical Sciences, 6(21), pp. 5012-5021, 2011.

https://doi.org/10.5897/IJPS11.694

[6] Gandomi, A. H., Kashani, A. R., Mousavi, M., Jalalvandi, M. "Slope stability analysis using evolutionary optimization techniques", International Journal for Numerical and Analytical Methods in Geomechanics, 41(2), pp. 251-264, 2017. https://doi.org/10.1002/nag.2554

[7] Kashani, A. R., Gandomi, A. H., Mousavi, M. "Imperialistic competitive algorithm: a metaheuristic algorithm for locating the critical slip surface in 2-dimensional soil slopes", Geoscience Frontiers, 7(1), pp. 83-89, 2016. https://doi.org/10.1016/j.gsf.2014.11.005

[8] Kaveh, A., Seddighian, M. R. "Optimization of Slope Critical Surfaces Considering Seepage and Seismic Effects Using Finite Element Method and Five Meta-Heuristic Algorithms", Periodica Polytechnica Civil Engineering, 65(2), pp. 425-436, 2021. https://doi.org/10.3311/PPci.17098 developed algorithms. As per the results and finding, CTSA-PS has demonstrated strongly competitive results for most of the benchmark functions and outperform the standard TSA and also other algorithms in a statistically significant manner. The new CTSA-PS algorithm has been successfully applied for seismic evaluation of earth slope and its effectiveness investigated through two numerical experiments. The results demonstrate that the proposed scheme outperforms the other methods in terms of better optimal solutions and could provide lower values of FOS and critical failure surfaces.

[9] Khajehzadeh, M., Taha, M. R., Eslami, M. "Multi-objective optimization of foundation using global-local gravitational search algorithm", Structural Engineering and Mechanics, 50(3), pp. 257-273, 2014.

https://doi.org/10.12989/sem.2014.50.3.257

[10] Marano, G. C., Quaranta, G., Monti, G. "Modified genetic algorithm for the dynamic identification of structural systems using incomplete measurements", Computer-Aided Civil and Infrastructure Engineering, 26(2), pp. 92-110, 2011. https://doi.org/10.1111/j.1467-8667.2010.00659.x

[11] Cheng, Y.-M. "Modified harmony methods for slope stability problems", In: Geem, Z. W. (ed.) Music-Inspired Harmony Search Algorithm, Springer, Berlin Heidelberg, Germany, 2009, pp. $141-162$. https://doi.org/10.1007/978-3-642-00185-7_9

[12] Zhang, K., Cao, P. "Modified electromagnetism-like algorithm and its application to slope stability analysis", Journal of Central South University of Technology, 18(6), pp. 2100-2107, 2011. https://doi.org/10.1007/s11771-011-0949-2

[13] Eslami, M., Shareef, H., Mohamed, A. "Optimization and coordination of damping controls for optimal oscillations damping in multi-machine power system", International Review of Electrical Engineering, 6(4), pp. 1984-1993, 2011.

[14] Khajehzadeh, M., Taha, M. R., Eslami, M. "A new hybrid firefly algorithm for foundation optimization", National Academy Science Letters, 36(3), pp. 279-288, 2013. https://doi.org/10.1007/s40009-013-0129-z

[15] Kaur, S., Awasthi, L. K., Sangal, A. L., Dhiman, G. "Tunicate swarm algorithm: a new bio-inspired based metaheuristic paradigm for global optimization", Engineering Applications of Artificial Intelligence, 90, Article number: 103541, 2020. https://doi.org/10.1016/j.engappai.2020.103541

[16] Zhu, D. Y., Lee, C. F., Qian, Q. H., Chen, G. R. "A concise algorithm for computing the factor of safety using the Morgenstern-Price method", Canadian Geotechnical Journal, 42(1), pp. 272-278, 2005. https://doi.org/10.1139/t04-072

[17] Duncan, J. M. "State of the art: limit equilibrium and finite-element analysis of slopes", Journal of Geotechnical Engineering, 122(7), pp. 577-596, 1996.

https://doi.org/10.1061/(ASCE)0733-9410(1996)122:7(577) 
[18] Fredlund, D. G., Krahn, J. "Comparison of slope stability methods of analysis", Canadian Geotechnical Journal, 14(3), pp. 429-439, 1977. https://doi.org/10.1139/t77-045

[19] Fellenius, W. "Calculation of the stability of earth dams", In: Proceedings of the Second Congress of Large Dams, Washington, DC, USA, 1936, pp. 445-463.

[20] Bishop, A. W. "The use of the slip circle in the stability analysis of earth slopes", Géotechinque, 5(1), pp. 7-17, 1955. https://doi.org/10.1680/geot.1955.5.1.7

[21] Spencer, E. "A method of analysis of the stability of embankments assuming parallel inter-slice forces", Géotechnique, 17(1), pp. $11-26,1967$.

https://doi.org/10.1680/geot.1967.17.1.11

[22] Morgenstern, N. R., Price, V. E. "The analysis of the stability of general slip surfaces", Géotechinque, 15(1), pp. 79-93, 1965. https://doi.org/10.1680/geot.1965.15.1.79

[23] Cheng, Y. M. "Location of critical failure surface and some further studies on slope stability analysis", Computers and Geotechnics, 30(3), pp. 255-267, 2003.

https://doi.org/10.1016/S0266-352X(03)00012-0
[24] Torczon, V. "On the convergence of pattern search algorithms", SIAM Journal on Optimization, 7(1), pp. 1-25, 1997. https://doi.org/10.1137/S1052623493250780

[25] Cherki, I., Chaker, A., Djidar, Z., Khalfallah, N., Benzergua, F. "A Sequential Hybridization of Genetic Algorithm and Particle Swarm Optimization for the Optimal Reactive Power Flow", Sustainability, 11(14), Article number: 3862, 2019 https://doi.org/10.3390/su11143862

[26] Mirjalili, S. "SCA: a sine cosine algorithm for solving optimization problems", Knowledge-Based Systems, 96, pp. 120-133, 2016. https://doi.org/10.1016/j.knosys.2015.12.022

[27] Mirjalili, S., Mirjalili, S. M., Lewis, A. "Grey wolf optimizer", Advances in Engineering Software, 69, pp. 46-61, 2014. https://doi.org/10.1016/j.advengsoft.2013.12.007

[28] Himanshu, N., Burman, A. "Determination of critical failure surface of slopes using particle swarm optimization technique considering seepage and seismic loading", Geotechnical and Geological Engineering, 37(3), pp. 1261-1281, 2019. https://doi.org/10.1007/s10706-018-0683-8 